\title{
Uptake and Detoxification of Organic Micropollutants by Macrophytes in Constructed Wetlands
}

\author{
A. Dordio ${ }^{\mathrm{a}, \mathrm{b}^{*}}$, A. J. P. Carvalho ${ }^{\mathrm{a}, \mathrm{c}}$, M. Hijosa-Valsero ${ }^{\mathrm{d}, \mathrm{e}}$, E. Becares $^{\mathrm{e}}$ \\ ${ }^{a}$ Chemistry Department, School of Sciences and Technology, University of Évora, Évora, \\ Portugal. \\ ${ }^{b}$ MARE - Marine and Environmental Research Centre, University of Évora, Évora, Portugal. \\ ${ }^{c} C Q E-E$ Evora Chemistry Centre, University of Évora, Évora, Portugal. \\ ${ }^{d}$ Center of Biofuels and Bioproducts, Agrarian Technological Institute of Castilla and León \\ (ITACyL), Villarejo de Orbigo, 24358 León, Spain. \\ ${ }^{e}$ University of León, Department of Biodiversity and Environmental Management, Faculty of \\ Biological Sciences, 24071 León, Spain
}

*Corresponding author

\begin{abstract}
Pollution by organic micropollutants (OMPs) is recognized worldwide as an emerging environmental problem. Since conventional wastewater treatment processes are generally ineffective for most of these pollutants, alternative/complementary technologies need to be considered with the aim of more efficiently addressing this problem. Constructed wetlands is a promising technology that uses plants and microorganisms to remove OMPs from wastewaters. As result of their sedentary nature, plants have evolved diverse abilities for dealing with toxic compounds present in their environment. They, therefore, possess a variety of pollutant attenuation mechanisms that makes their use in wastewater treatment more feasible than physical and chemical processes.

This chapter presents an overview of OMPs' fate inside plants, following their uptake, and discusses the main factors influencing their uptake and translocation within plants. The various phases of the metabolic transformations of organic pollutants inside plants, which have the aim of modifying the xenobiotics in order to make them less toxic to the plants, are also described in this chapter. Finally, a brief account is given on the response of antioxidant defense systems of
\end{abstract}


plants which are triggered by abiotic stress conditions that may result from long-term exposure of plants to OMPs. 